\title{
Linfoma $\mathbf{T}$ intestinal epiteliotrópico monomórfico: Reporte de caso.
}

\begin{abstract}
*Correspondencia:
pazvinti18@hotmail.com

Atahualpa Chávez González Mz P3 V6, Guayaquil 090514, Departamento de Anatomía Patológica de SOLCA, Guayaquil, Ecuador. Teléfono [593] 0998822184
\end{abstract}

Conflicto de intereses: Los autores declaran no tener conflictos de intereses.

Recibido: 22 de Mayo 2021

Aceptado: 28 de Julio, 2021

Publicado: 33 de Agosto, 2021

Editor: Dr. Felipe Campoverde

Membrete bibliográfico: Vintimilla $\mathrm{M}$, Yoncón $\mathrm{R}$, Ordinola $\mathrm{C}$, Nieto J, Ochoa A, Human F. Linfoma $T$ intestinal epiteliotrópico monomórfico: Reporte de caso. Rev. Oncol. Ecu 2021;31(2):114121.

ISSN: 2661-6653

DOI: https://doi.org/10.33821/558

Copyright Vintimilla M, et al. Este artículo es distribuido bajo los términos de Creative Commons Attribution License, el cual permite el uso y redistribución citando la fuente y al autor original.

\section{Monomorphic epitheliotropic intestinal T Iymphoma:} A case report.

\section{María Paz Vintimilla Cazorla*1, Ricardo Yoncón Romero2 ${ }^{\mathbb{D}}$, Carlos Be- nites Ordinola1, Jorge Nieto Orellana1, Anthony Ochoa Bastidas1, Fuad Huamán Garaicoa ${ }^{1,3}$ (D)}

1. SOLCA - Guayaquil.

2. Postgrado de Anatomía Patológica, Universidad Central del Ecuador.

3. Carrera de Medicina, Universidad Católica de Santiago de Guayaquil.

\section{Resumen}

Introducción: El linfoma de células T intestinal epiteliotrópico monomórfico es una neoplasia derivada de linfocitos intraepiteliales. La etiología es desconocida, pero se ha asociado a enfermedad celíaca o a síndrome de malabsorción, aunque en la actualidad se considera un linfoma esporádico. La histopatología se caracteriza por linfocitos de tamaño intermedio a pequeño, con mínimo pleomorfismo, que generalmente expresan CD8 y CD56.

Reporte de caso: Mujer de 60 años con dispepsia de larga evolución, fue ingresada por dolor abdominal agudo, diarrea, nausea, vómito bilioso y pérdida de peso. En los estudios se determinó anemia microcítica hipocrómica y desequilibrio hidroelectrolítico. Se evidenció por tomografía de abdomen una lesión nodular esplénica y engrosamiento concéntrico segmentario de yeyuno e íleon distal asociado a la presencia de líquido libre en cavidad abdominal, se consideró una masa tumoral.

Evolución: Se realizó una laparotomía exploratoria con resección de yeyuno-transverso, en patología se determinó la presencia de 3 tumores: a nivel de yeyuno de $16 \times 8.5 \times 6 \mathrm{~cm}$, en el íleon distal de $13 \times 6.5 \times 3$ $\mathrm{cm}$ y en el ciego de $5 \times 4 \times 2 \mathrm{~cm}$ con histología de linfoma no Hodgkin $T$ de alto grado, intestinal primario, de tipo monomórfico epiteliotrópico (MEITL). Fue dada de alta diez días después. Dos semanas luego desarrollo sepsis de foco urinario, deshidratación, síndrome de intestino corto, hiperamonemia y neumonía de focos múltiples con derrame pleural bilateral. Falleció al sexto día de hospitalización.

Conclusiones: El MEITL es una neoplasia agresiva primaria intestinal de reciente identificación y pobre pronóstico. No se conocen en su totalidad los mecanismos moleculares asociados a esta entidad. El caso presentado demostró un curso clínico similar al reportado en la literatura.

Palabras claves:

DeCS: Linfoma de Células B de la Zona Marginal, Linfoma de Células T, Neoplasias Intestinales, Intestino Delgado, Informes de casos.

DOI: $10.33821 / 558$ 


\begin{abstract}
Introduction: Monomorphic epitheliotropic intestinal T-cell lymphoma is a derived neoplasia of intraepithelial lymphocytes. The etiology is unknown, but it has been associated with celiac disease or malabsorption syndrome, although it is currently considered a sporadic lymphoma. Histopathology is characterized by medium to small lymphocytes with minimal pleo-morphism, which generally express CD8 and CD56.

Case report: A 60-year-old woman with long-standing dyspepsia was admitted for acute abdominal pain, diarrhea, nausea, bilious vomiting, and weight loss. In the studies, hypochromic microcytic anemia and fluid and electrolyte imbalance were determined. A splenic nodular lesion and segmental concentric thickening of the jejunum and distal ileum associated with the presence of free fluid in the abdominal cavity was evidenced by abdominal tomography, it was considered a tumor mass.

Evolution: An exploratory laparotomy was performed with transverse jejunum resection, in pathology the presence of 3 tumors was determined: at the jejunum level of $16 \times 8.5 \times 6 \mathrm{~cm}$, in the distal ileum of $13 \times 6.5 \times 3$ $\mathrm{cm}$ and in the cecum of $5 \times 4 \times 2 \mathrm{~cm}$ with histology of high-grade non-Hodgkin T lymphoma, primary intestinal, epitheliotropic monomorphic type (MEITL). She was discharged ten days later. Two weeks later, she developed sepsis with a urinary focus, dehydration, short bowel syndrome, hyper-amonemia, and multiple-focus pneumonia with bilateral pleural effusion. He died on the sixth day of hospitalization.
\end{abstract}

Conclusions: MEITL is a recently identified aggressive primary intestinal neoplasm and poor prognosis. The molecular mechanisms associated with this entity are not fully known. The case presented showed a clinical course similar to that reported in the literature.

Keywords: MESH: Lymphoma, B-Cell, Marginal Zone; Lymphoma, T-Cell; Intestinal Neoplasms; Intestine, Small; Case Reports.

DOI: $10.33821 / 558$

\title{
Introducción
}

Los linfomas extranodales gastrointestinales representan del 1\% al $4 \%$ de los tumores del tracto digestivo y la afectación por linfoma periférico de células T es relativamente rara, siendo más frecuente el de células B [1-2].

El linfoma de células T intestinal epiteliotrópico monomórfico (MEITL, por sus siglas en inglés) es una entidad provisional en la clasificación de la OMS de 2017, conocido hasta la clasificación de 2008 como linfoma de células T asociado a enteropatía de tipo II (EATL). Es una neoplasia derivada de linfocitos intraepiteliales (LIEs), de etiología desconocida. Aunque en reportes lo han asociado a enfermedad celíaca o a síndromes de malabsorción, en la actualidad se considera un linfoma esporádico [ㄱ-7] 
La histopatología se caracteriza por una proliferación de elementos linfoide de tamaño intermedio a pequeño, con mínimo pleomorfismo. Estos linfocitos generalmente expresan CD8 y CD56, marcadores que sugieren un origen T gamma-delta [8].

Clínicamente, esta neoplasia se considera muy agresiva y tiene pobre pronóstico. Si la enfermedad no es tratada, los pacientes afectados fallecerán en los meses posteriores a su diagnóstico, debido a perforación multifocal intestinal o por sangrado. El tratamiento consiste en cirugía, quimioterapia o trasplante de células madre [8].

\section{Caso Clínico}

\section{Descripción del caso}

Paciente mujer de 60 años de edad, de procedencia y residencia en la provincia del GuayasEcuador, con antecedentes personales de alergia a las fabáceas y pancreatitis aguda diagnosticada hace 20 años. Presenta un cuadro de dispepsia de larga evolución. Dos días antes del ingreso presentó dolor abdominal, de gran intensidad, tipo cólico, a nivel de mesogastrio, que se irradiaba en hemicinturón; además se acompañó con deposiciones diarreicas -en número de 10 diarias-, náusea que llegó al vómito por múltiples ocasiones de tipo biliosas y pérdida de peso. Al examen físico mostró facies álgica, mucosas orales secas, abdomen distendido, doloroso a la palpación a nivel de epigastrio y mesogastrio, ruidos hidroaéreos disminuidos.

\section{Taller diagnóstico}

En el hemograma y la química sanguínea se identificó anemia microcítica hipocrómica y desequilibrio hidroelectrolítico. Se solicitó una tomografía computarizada de abdomen donde se evidenció la presencia de una lesión nodular esplénica y engrosamiento concéntrico segmentario de yeyuno e íleon distal asociado a la presencia de líquido libre en cavidad abdominal. El estudio colonoscópico no demostró alteraciones macroscópicas de la mucosa.

\section{Evolución}

Se decide intervención quirúrgica por sospecha de lesión tumoral, laparotomía exploratoria más resección de yeyuno-transverso anastomosis latero-lateral. Se remite la pieza quirúrgica al Departamento de Anatomía Patológica, donde se demuestra una primera lesión tumoral a nivel de yeyuno de $16 \times 8.5 \times 6 \mathrm{~cm}$, una segunda lesión en el íleon distal de $13 \times 6.5 \times 3 \mathrm{~cm}$ y una tercera lesión en el ciego de 5x4×2cm. Los hallazgos morfológicos e inmunofenotípicos (Fig. 1) corresponden a un linfoma no Hodgkin $T$ de alto grado, intestinal primario, vinculable al tipo monomórfico epiteliotrópico (MEITL). Fue dada de alta en mejores condiciones diez días después.

Después de dos semanas acudió por el área de emergencia, taquicárdica, hipotensa, desorientada y con mala ventilación mecánica. Es trasladada a la Unidad de Cuidados Intensivos, por presentar shock séptico de foco urinario, deshidratación por síndrome de intestino corto, hiperamonemia y neumonía de focos múltiples con derrame pleural bilateral. Se solicitó valoración de clonalidad gamma delta: esta no se logró realizar pues la paciente presentó evolución clínica desfavorable. Falleció al sexto día de hospitalización. 


\section{Discusión}

El sitio primario del MEITL ocurre en un 80 a un $84 \%$ a nivel de intestino delgado, con predominio en el sexo masculino y en poblaciones de origen asiático. La media de supervivencia es de siete meses después del diagnóstico. Es considerada como una entidad muy agresiva. Debido a la rareza de esta patología y su identificación relativamente reciente, la incidencia, el curso natural y el pronóstico clínico del MEITL no están bien dilucidados [ㄴ, 으-10].

En el estudio de Yi y cols. se observó que el sitio de aparición más frecuente de lesión tumoral fue el yeyuno [1]. El de la paciente analizada se encontró a nivel de yeyuno y de ciego. Además, se identificó una persistente proliferación de células tumorales a nivel de la mucosa que macroscópicamente se veía conservada, lo que fue reportado en el trabajo de Aoyama y cols.[ 7] Tian y cols., reportan dos casos de MEITL donde inicialmente se había considerado una colitis ulcerosa y una colitis linfocítica respectivamente, con clínica de dolor abdominal y deposiciones diarreicas. La colonoscopía mostró hiperemia y ulceraciones [11]. El problema surge en la presentación de hallazgos inespecíficos colonoscópicos, en la dificultad de ofrecer un diagnóstico oportuno, retrasando el tratamiento y conllevando a un peor pronóstico.

Los hallazgos morfológicos e inmunofenotípicos son necesarios para el diagnóstico final. En primera instancia se solicitan pruebas para identificar su linaje celular (B o T/NK): en nuestro caso al ser analizado se obtuvo CD20-, CD3+, CD7+, CD56+, presentó pérdida de la expresión de CD5. Las células T malignas, a pesar de la expresión de CD3 y CD7 reportada aquí, suelen perder expresión de uno o varios de los marcadores de linaje T4.

En la inmunohistoquímica se evidencia positividad para los marcadores CD3, CD8, CD56 y granzima B en un 63\%, negatividad para CD5 y CD30. Un 9\% de casos expresan CD4 junto con CD8. Un 80\% muestra rearreglos del receptor TCR-y y TCR- $89[11,12]$.

En la mayoría de MEITL se presenta un inmunofenotipo citotóxico distintivo en el cual los marcadores inmunohistoquímicos TIA1, CD3, CD7, CD8 y CD56 se encuentran positivos, tal como se identifica en esta paciente. Además, el marcador CD56 cumple un papel fundamental en el diagnóstico, pues en el MEITL es positivo en el 73\% de los casos, pero negativo en el EATL. Por esta razón, en la clasificación de linfomas de la Organización Mundial de la Salud (OMS), hasta 2010 se lo denominaba linfoma T intestinal monomórfico CD56+ [4, 8].

En el MEITL el inmunomarcador CD8 es positivo y en el EATL es negativo. En el caso presentado, este marcador es positivo, y se acompaña en un menor grado de positividad CD4; hallazgo que está en concordancia con la literatura analizada $[4, \underline{9}, 11]$. 


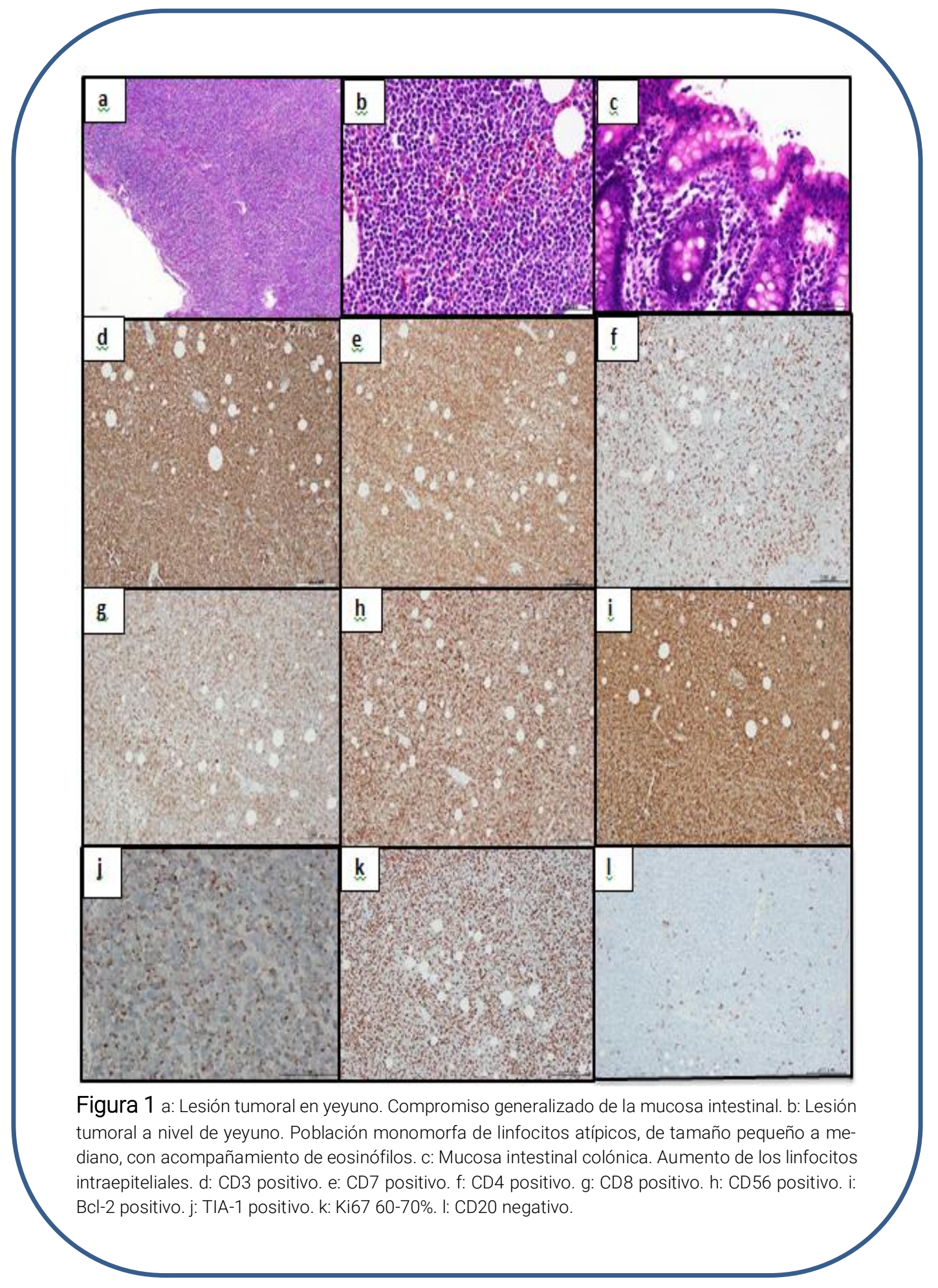




\section{Conclusiones}

El MEITL es una entidad nueva e infrecuente, posee muchas similitudes en la presentación y el curso clínico con el EATL pero no comparten todas las alteraciones inmunofenotípicas. Por la agresividad de esta patología, la mayoría de los pacientes fallecen al poco tiempo de su diagnóstico. Los hallazgos colonoscópicos pueden ser inespecíficos e incluso dar el aspecto de una mucosa conservada. Es fundamental la sospecha clínica y el diagnóstico histológico con análisis inmunohistoquímico, para un tratamiento oportuno.

\section{Agradecimientos}

Nota del Editor

La Revista Oncología Ecu

permanece neutral con res-

pecto a los reclamos juris-

diccionales en mapas publicados y afiliaciones institucionales.
Los autores agradecen a todas las personas de la Institución que colaboraron en el desarrollo de esta investigación.

\section{Información administrativa}

\section{Abreviaturas}

EATL: linfoma de células T asociado a enteropatía de tipo II

MEITL: Linfoma de células T intestinal epiteliotrópico monomórfico.

\section{Archivos Adicionales}

Ninguno declarado por los autores.

\section{Fondos}

Los autores no recibieron ningún tipo de reconocimiento económico por este trabajo de investigación. Los gastos producidos en la investigación fueron asumidos por los investigadores.

\section{Disponibilidad de datos y materiales}

Existe la disponibilidad de datos bajo solicitud al autor de correspondencia. No se reportan otros materiales.

\section{Contribuciones de los autores}

María Paz Vintimilla Cazorla: realizó la conceptualización, metodología, administración del proyecto, curación de datos, análisis formal, adquisición de fondos, investigación, recursos, software, redacción - borrador original. Ricardo Yoncón Romero: realizó la conceptualización, investigación y redacción -borrador original.

Fuad Huamán Garaicoa: conceptualización, validación, redacción -borrador original. Carlos Benites Ordinola: validación, escritura: revisión y edición.

Jorge Nieto Orellana: validación, visualización, supervisión, escritura: revisión y edición. 
Antony Ochoa Bastidas: validación, visualización, supervisión, escritura: revisión y edición. Todos los autores leyeron y aprobaron la versión final del manuscrito.

Aprobación del comité de ética

No requerido para casos clínicos.

Consentimiento para publicación

El presente estudio cuenta con la aprobación y permiso de publicación escrito por parte de los familiares del paciente.

\section{Referencias}

1. Yi JH, Lee GW, Do YR, Jung HR, Hong JY, Yoon DH, Suh C, et al. Multicenter retrospective analysis of the clinicopathologic features of monomorphic epitheliotropic intestinal T-cell lymphoma. Ann Hematol. 2019 Nov;98(11):2541-2550. doi: 10.1007/s00277-019-03791-y. Epub 2019 Sep 6. PMID: 31493002.

2. Lizarzaburu V, Miñano C, Caballero J, Vásquez C, Castro E. Linfoma gástrico no Hodgkin perforado. Rev. gastroenterol. Perú 2017;37(3):271-274. SU: scielo.pe/S1022

3. Ishibashi H, Nimura S, Kayashima Y, Takamatsu Y, Iwasaki H, Harada N, Momosaki S, Takedatsu H, Sakisaka S, Takeshita M. Endoscopic and clinicopathological characteristics of gastrointestinal adult T-cell leukemia/lymphoma. J Gastrointest Oncol. 2019 Aug;10(4):723-733. doi 10.21037/jgo.2019.03.03. PMID: 31392053; PMCID: PMC6657316.

4. Bhagat G, Isaacson PG. Enteropathy-Associated T-Cell Lymphoma and Other Primary Intestinal T-Cell Lymphomas. En: Jaffe ES, Arber DA, Campo E, Harris NL, Quintanilla-Martinez L. Hematopathology. 2 ed. Elsevier, Philadelphia, EE.UU. 2017;2(38) 693-711.

5. Goldblum JR. Large Bowel. En: Goldblum JR, Lamps LW, McKenney JK, Myers JL. Rosai and Ackerman's Surgical Pathology. 11 ed. Elsevier, Philadelphia, EE.UU. 2016;17(10) 686.

6. Lenti MV, Biagi F, Lucioni M, Di Sabatino A, Paulli M, Corazza GR. Two cases of monomorphic epitheliotropic intestinal T-cell lymphoma associated with coeliac disease. Scand J Gastroenterol. 2019 Aug;54(8):965-968. doi: 10.1080/00365521.2019.1647455. Epub 2019 Jul 30. PMID: 31361171.

7. Aoyama $Y$, Tsunemine H, Zushi $Y$, Maruoka H, Goto Y, Kodaka T, et al. Colonal monomorphic epitheliotropic intestinal T-cell lymphoma with novel phenotype of cytoplasmic CD3 expression. J Clin Exp Hematop. 2018 Jul 11;58(2):102-106. doi: 10.3960/jslrt.18002. Epub 2018 Apr 13. PMID: 29657256; PMCID: PMC6413146.

8. Samuel R, Krill T, Merwat S. Monomorphic Epitheliotropic Intestinal T cell Lymphoma: a Rare Cause of Chronic Diarrhea. J Gastrointest Cancer. 2019 Dec;50(4):1051-1054. doi: 10.1007/s12029-01900210-3. PMID: $\underline{30761475}$.

9. Liu Y, Li YH, Li J, Qian JM, Zhou WX, Luo YP. Monomorphic epitheliotropic intestinal T-cell lymphoma complicated by common variable immunodeficiency. Intest Res. 2017 Oct;15(4):546-547. doi: 10.5217/ir.2017.15.4.546. Epub 2017 Oct 23. PMID: 29142526; PMCID: PMC5683989. 
10. van Vliet C, Spagnolo DV. T- and NK-cell lymphoproliferative disorders of the gastrointestinal tract: review and update. Pathology. 2020 Jan;52(1):128-141. doi: 10.1016/j.pathol.2019.10.001. Epub 2019 Nov 11. PMID: 31727264.

11. Tian S, Xiao SY, Chen Q, Liu H, Ping J. Monomorphic epitheliotropic intestinal T-cell lymphoma may mimic intestinal inflammatory disorders. Int J Immunopathol Pharmacol. 2019 JanDec;33:2058738419829387. doi: 10.1177/2058738419829387. PMID: 30757928; PMCID: PMC6376542.

12. Ishibashi H, Nimura S, Kayashima Y, Takamatsu Y, Aoyagi K, Harada N, et al. Multiple lesions of gastrointestinal tract invasion by monomorphic epitheliotropic intestinal T-cell lymphoma, accompanied by duodenal and intestinal enteropathy-like lesions and microscopic lymphocytic proctocolitis: a case series. Diagn Pathol. 2016 Jul 25;11(1):66. doi: 10.1186/s13000-016-0519-x. PMID: 27457239; PMCID: PMC4960898. 